\title{
Ultra low-dose single photon emission computed tomography myocardial perfusion imaging
}

\author{
William B. Nelson 1, 2, 3, Chad M. House ${ }^{1,2}$, Patricia K. Turnquist ${ }^{2,4}$, Jenny M. Spence ${ }^{2,4}$, Mary A. \\ Anstadt ${ }^{1,2}$, Glenn A. Nickele ${ }^{1,2}$, Katie A. Moriarty ${ }^{1,2}$, Charles K. Stone ${ }^{5}$ \\ 1. Department of Cardiology, Regions Hospital, Minnesota, USA. 2. HealthPartners Medical Group, Los Angeles, USA. 3. \\ School of Medicine, University of Minnesota, Minnesota, USA. 4. Department of Nuclear Medicine, Regions Hospital, \\ Minnesota, USA. 5. Cardiovascular Medicine Division, University of Wisconsin, Madison, USA.
}

Correspondence: Chad House. Address: Mail Stop 11102M, 640 Jackson Street, St. Paul, MN 55101, Minnesota, Minnesota, USA. Email: chad.m.house@healthpartners.com

Received: February 12, 2014

DOI : $10.5430 /$ ijdi.v1n2p54
Accepted: March 2, $2014 \quad$ Online Published: April 22, 2014

URL: http://dx.doi.org/10.5430/ijdi.v1n2p54

\section{Abstract}

Background: Single photon emission computed tomography (SPECT) myocardial perfusion imaging (MPI) provides excellent diagnostic and prognostic information. A primary limitation of SPECT is radiation exposure. New cadmium zinc telluride (CZT) technology has allowed for reduced radiation exposure.

Methods: A cohort of 414 consecutive patients undergoing very low-dose SPECT MPI utilizing CZT technology was analyzed. Patients received rest/stress dosing strategies of 111/333, 148/444, 222/666, 296/888, 370/1110 Megabecquerels (MBq) (3/9, 4/12, 6/18, 8/24, 10/30 millicuries) depending on their weight and body mass index. Image quality was visually assessed by the reading cardiologist (scale: 1 = poor; 2 = fair; 3 = good; 4 = excellent).

Results: $58 \%$ of patients received $111 / 333 \mathrm{MBq}, 22 \%$ received $148 / 444 \mathrm{MBq}, 13 \%$ received 222/666MBq, $6 \%$ received 296/888MBq, and 1\% received 370/1110MBq. The estimated radiation dose was 3.6, 4.8, 7.2, 9.6, and 12 millisieverts respectively. The average image quality for the cohort was $3.3 \pm 0.7$. The rest/stress counts $\left(10^{3}\right)$ for $111 / 333$ and $148 / 444$ groups were $532 \pm 212 / 1718 \pm 525$ and $545 \pm 177 / 1748 \pm 399$, while the rest/stress counts $\left(10^{3}\right)$ for the 222/666 and 296/888 groups were $673 \pm 178 / 2235 \pm 923$ and $764 \pm 249 / 2588 \pm 788$ respectively. The 111/333 and 148/444 groups had significantly lower counts than the 222/666 and 296/888 groups, but only the 296/888 group had a significant difference in image quality when compared to the 111/333 group, with the 111/333 group having better image quality.

Conclusion: CZT technology permits rapid, very low-dose MPI studies to be performed, thus, reducing the radiation exposure for patients, while still providing high quality, diagnostic perfusion results. The majority of our patients were imaged at less than $4 \mathrm{mSv}$.

\section{Keywords}

Myocardial perfusion imaging, Dose reduction, Cadmium zinc telluride

\section{I ntroduction}

Single photon emission computed tomography (SPECT) myocardial perfusion imaging (MPI) has good sensitivity and specificity for detecting ischemia or infarction. It is widely available, making it an excellent modality to evaluate for the 
presence and significance of obstructive coronary artery disease ${ }^{[1]}$. In addition to being a useful diagnostic tool, it also provides prognostic information, for example, normal exercise perfusion is associated with a very low rate of subsequent cardiac events ${ }^{[2]}$.

A primary limitation of SPECT MPI is the associated radiation levels, which traditionally range between 10 and 20 millisieverts $(\mathrm{mSv})^{[3]}$. New cadmium zinc telluride (CZT) imaging technology measures higher photon flux compared to standard sodium-iodide scintillation technology. It has been shown to provide excellent diagnostic quality images, while allowing for reduce isotope dosage and shorter imaging acquisition times ${ }^{[4-6]}$. Given the concern over radiation dose in medical imaging, our goal was to reduce radiation dose for patients requiring nuclear stress testing, while still maintaining rapid, high quality diagnostic and prognostic imaging.

\section{Methods}

A prospective observational study was conducted on patients undergoing MPI between 11/16/2011 and 1/31/2012. The institutional review board approved this research project, with waiver of informed consent. All studies were performed in the ICANL accredited nuclear imaging lab at Regions Hospital and all studies were interpreted by a cardiologist who has been board certified in nuclear cardiology.

Collected data included: age, gender, rest/stress dose [Megabecquerels (MBq) and millicuries (mCi)], rest/stress counts, image quality, weight, body mass index (BMI), history of coronary artery disease, diabetes mellitus, hypertension, hyperlipidemia, smoking, perfusion results, and cardiac catherization results if performed. Photon counts were representative of the entire field of view. Image quality was subjectively assessed by the reading cardiologist (8 total) according to a 4-point scale of excellent, good, fair, or poor. Studies were interpreted in a semi-quantitative fashion, with cardiologists assessing them visually and, as needed, incorporating the sum stress score and computer models of infarct and/or ischemia. Attenuation correction was not used. In certain patients, prone imaging was obtained to limit sub-diaphragmatic and diaphragmatic attenuation.

The software on the CZT camera allows for comparison of photon counts and image reconstruction at different imaging times. We initially used the imaging times and isotope dosages reported by Duvall et al ${ }^{[6]}$. We titrated our protocol to reduced isotope dosages and determine optimal imaging times, in order to maintain adequate photon counts and high quality images, arriving at the isotope dosages and imaging times utilized in this study.

All patients were dosed according to the protocol seen in Table 1, with an allowed variance of $\pm 10 \%$. Nuclear imaging was performed on a General Electric (GE) Discovery NM 530c (GE Healthcare, Haifa, Israel) nuclear cardiology imaging system, with focused multi-pinhole collimation, which improves sensitivity and detection efficiency. Rest/stress scan image acquisition times were 6.5 and 4.5 minutes for all groups. The radiopharmaceutical utilized was Tc-99m Sestamibi.

Table 1. Dosing Scheme

\begin{tabular}{lcc}
\hline$<200$ lbs. & $3 \mathrm{mCi}$ & $9 \mathrm{mCi}$ \\
$\begin{array}{l}<M I<32 \\
<200 \text { lbs. }\end{array}$ & $4 \mathrm{mCi}$ & $12 \mathrm{mCi}$ \\
$\begin{array}{l}\text { BMI } 33-40 \\
200-250 \text { lbs. } \\
\text { BMI }<32\end{array}$ & $4 \mathrm{mCi}$ & $12 \mathrm{mCi}$ \\
$200-250$ lbs. & $6 \mathrm{mCi}$ & $18 \mathrm{mCi}$ \\
BMI $33-40$ & $8 \mathrm{mCi}$ & $24 \mathrm{mCi}$ \\
$251-300$ lbs. & $10 \mathrm{mCi}$ & $30 \mathrm{mCi}$ \\
BMI $<40$ & \\
$301-349$ lbs. & & \\
BMI $<40$ & & \\
\hline
\end{tabular}




\subsection{Nuclear cardiology protocol}

Patients were instructed to fast for two hours prior to arriving for their MPI study. Patients undergoing pharmacologic stress were instructed to abstain from caffeine for 12 hours prior to arrival.

Upon arrival, intravenous access was obtained and the initial radiopharmaceutical dose was administered. Patients were then instructed to wait approximately ninety minutes before their resting image acquisition was performed, and to drink 1-2 glasses of water to reduce the impact of bowel interference. Resting image acquisition was for 6.5 minutes, with the patients lying supine and their arms positioned above their head.

Two hours post rest injection, the patient underwent a symptom limited exercise stress (Bruce or a modified Bruce protocol) or pharmacologic stress (adenosine, dobutamine, or regadenoson). The radiopharmaceutical was then injected according to one of the following parameters:

- $\quad$ 1-2 minutes before the anticipated termination of the exercise test

- $\quad 2$ minutes into a 4 minute adenosine infusion

- When heart rate was $\geqslant 85 \%$ of maximum predicted heart rate during dobutamine infusion

- 45 seconds after the administration of regadenoson

- $\quad$ As instructed by the exercise physiologist

Stress image acquisition was performed 15 minutes after exercise and dobutamine stress, and an hour after adenosine or regadenoson stress. The patients were scanned for 4.5 minutes supine, with gating, and for 4.5 minutes prone, without gating.

\subsection{Statistics}

Continuous variables are expressed as the mean \pm one standard deviation, with the remaining variables expressed as percentages. Comparisons of continuous and dichotomous variables were conducted using an unpaired t-test or Fischer's Exact Test, with $<0.05$ considered statistically significant. A weighted kappa coefficient was used to assess the inter-observer variability in image quality in $10 \%$ of the patients. A two-by-two table was setup to assess sensitivity, specificity, positive predictive value, negative predictive value, and accuracy in patients referred for coronary angiography after their SPECT MPI.

\section{Results}

There were 414 consecutive patients identified who underwent the low-dose MPI protocol using CZT technology. The group had an average age of $63 \pm 13$ years, an average weight of $188 \pm 41 \mathrm{lbs}$, an average BMI of $29 \pm 4.9 \mathrm{~kg} / \mathrm{m}^{2}$, and $58 \%$ (242/414) of the patients were male. The majority of patients underwent pharmacological stress testing, with 58\% (239/414) undergoing pharmacological stress and 42\% (175/414) exercise stress. Adenosine was the stress agent for 93\% (223/239) of pharmacologic studies, 5\% (11/239) received regadenoson, and 2\% (5/239) received dobutamine. A small percentage (8\%) (17/223) of the patients receiving adenosine walked at 1.0 mile per hour on the treadmill during their infusion to mitigate any symptoms associated with the stress agent. Additional patient characteristics are provided in Table 2. 
Table 2. Patient Characteristics

\begin{tabular}{|c|c|c|c|c|c|c|}
\hline & $\begin{array}{l}\text { Complete } \\
\text { Cohort }\end{array}$ & $\begin{array}{l}\text { 111/333MBq } \\
(3 / 9 m C i)\end{array}$ & $\begin{array}{l}\text { 148/444MBq } \\
(4 / 12 \mathrm{mCi})\end{array}$ & $\begin{array}{l}\text { 222/666MBq } \\
(6 / 18 \mathrm{mCi})\end{array}$ & $\begin{array}{l}\text { 296/888MBq } \\
(8 / 24 \mathrm{mCi})\end{array}$ & $\begin{array}{l}\text { 370/1110MBq } \\
(10 / 30 \mathrm{mCi})\end{array}$ \\
\hline $\begin{array}{l}\text { Number of } \\
\text { Patients }\end{array}$ & 414 & $\begin{array}{l}242 \\
(58 \%)\end{array}$ & $\begin{array}{l}91 \\
(22 \%)\end{array}$ & $\begin{array}{l}53 \\
(13 \%)\end{array}$ & $\begin{array}{l}25 \\
(6 \%)\end{array}$ & $\begin{array}{l}3 \\
(1 \%)\end{array}$ \\
\hline Female (\%) & 42 & 51 & 24 & 49 & 4 & 0 \\
\hline Age (years) & $63 \pm 13$ & $65 \pm 15$ & $60 \pm 13$ & $60 \pm 12$ & $56 \pm 10$ & $60 \pm 5$ \\
\hline Weight (lbs) & $188 \pm 41$ & $162 \pm 26$ & $208 \pm 17$ & $227 \pm 16$ & $263 \pm 12$ & $323 \pm 15$ \\
\hline BMI & $29 \pm 4.9$ & $26 \pm 3.5$ & $31 \pm 2.6$ & $36 \pm 2.2$ & $35 \pm 2.6$ & $37 \pm 1.5$ \\
\hline CAD (\%) & 42 & 44 & 32 & 49 & 44 & 33 \\
\hline DM (\%) & 30 & 24 & 27 & 49 & 52 & 33 \\
\hline HTN (\%) & 71 & 70 & 69 & 70 & 76 & 100 \\
\hline HLD (\%) & 68 & 66 & 66 & 70 & 88 & 33 \\
\hline $\begin{array}{l}\text { Current } \\
\text { Smoker (\%) }\end{array}$ & 19 & 21 & 20 & 11 & 12 & 0 \\
\hline
\end{tabular}

Of the 414 patients, 58\% (242/414) received 111/333MBq (3/9mCi), 22\% (91/414) received 148/444MBq (4/12mCi), $13 \%$ (53/414) received 222/666MBq (6/18mCi), 6\% (25/414) received 296/888MBq (8/24mCi), and the remaining $1 \%$ $(3 / 414)$ received $370 / 1110 \mathrm{MBq}(10 / 30 \mathrm{mCi})$ with average rest/stress doses for the whole group of $151.7 \pm 59.2 \mathrm{MBq}$ and $444 \pm 177.6 \mathrm{MBq}(4.1 \pm 1.6$ and $12 \pm 4.8 \mathrm{mCi})$. The estimated radiation dose was 3.6, 4.8, 7.2, 9.6, and 12mSv respectively (see Figure 1), with an average expected radiation dose for the whole group of $4.8 \mathrm{mSv}$.

\section{Expected Radiation Per Dosing Strategy}

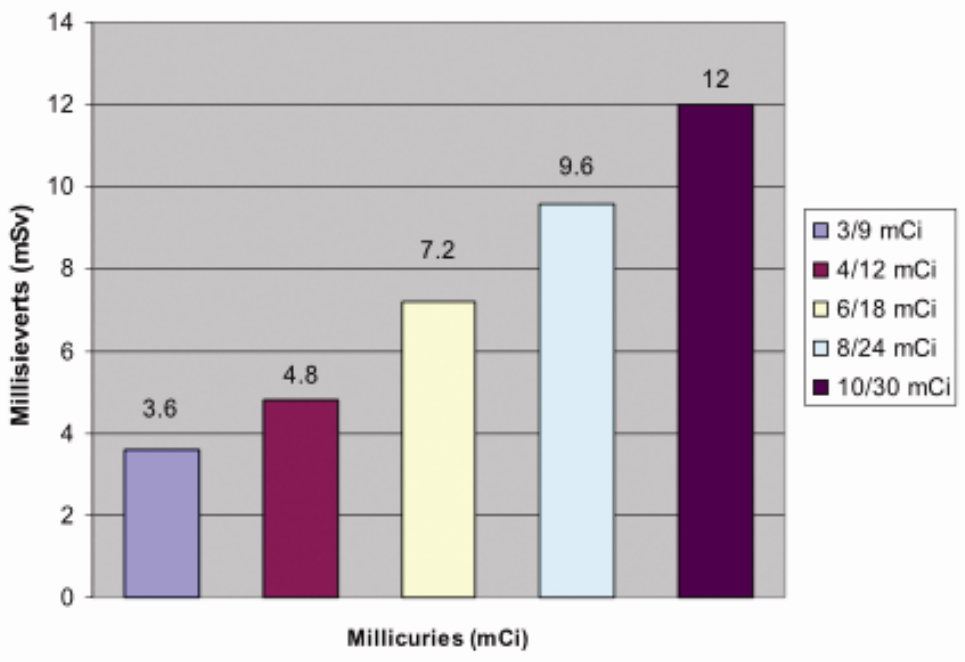

Figure 1. Expected Radiation Exposure by Dosing Strategy

The rest/stress count data for each group is shown in Figure 2a and 2b. The 222/666 and 296/888 groups had significantly more counts compared to the 111/333 and 148/444 groups. There was no significant difference in stress counts observed between the two stress methods. 


\section{Resting Count Comparison (Counts $10^{3}$ )}

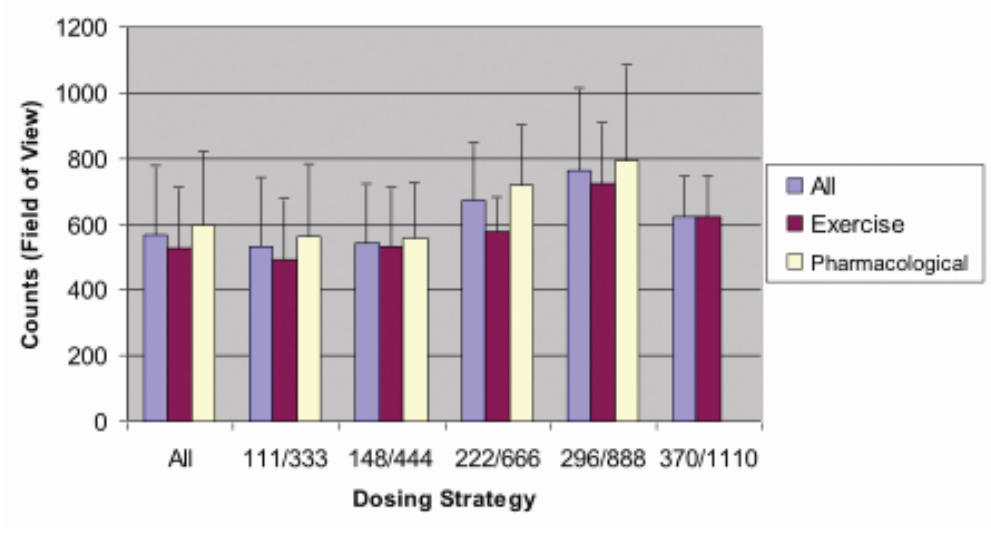

Figure 2a. Resting Count Comparison by Dosing Strategy

Figure 2a. Statistically significant differences include: All Patients: 111/333 vs. 222/666 and 296/888; $148 / 444$ vs. 222/666 and 296/888. Exercise Group: 111/333 vs. 296/888; 148/444 vs. 296/888; 222/666 vs. 296/888. Pharmacologic Group: $111 / 333$ vs. 222/666 and 296/888; $148 / 444$ vs. 222/666 and 296/888. Exercise vs. Pharmacologic Comparison:The whole exercise cohort vs. the whole pharmacologic cohort, along with the 111/333 and 222/666 exercise groups vs. the 111/333 and 222/666 pharmacologic groups.

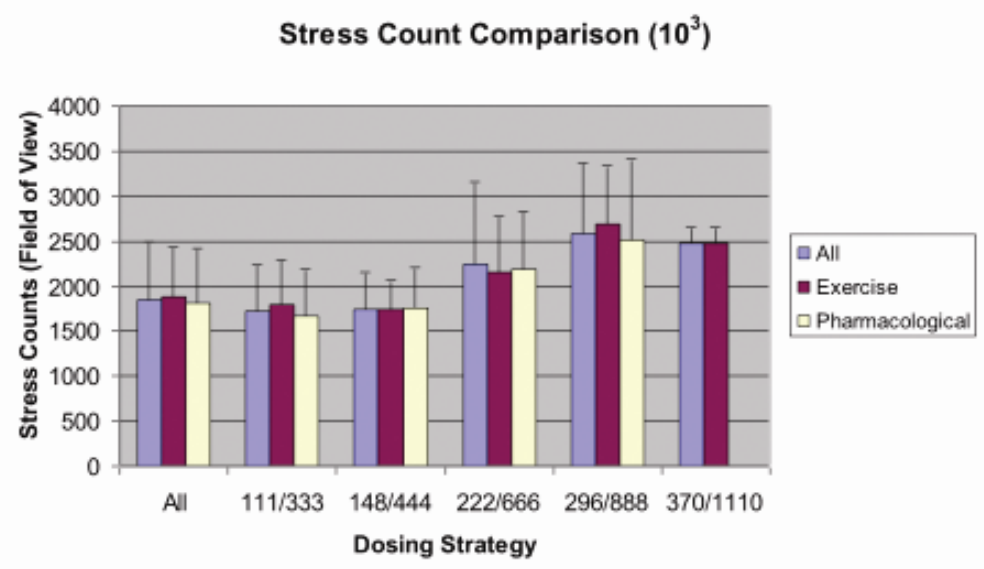

Figure 2b. Stress Count Comparison by Dosing Strategy

Figure 2b. Statistically significant differences include: All Patients: 111/333 vs. 222/666 and 296/888; 148/444 vs. 222/666 and 296/888. Exercise Group: 296/888 vs. 111/333, 148/444, and 222/666; 148/444 vs. 222/666. Pharmacologic Group: 148/444 vs. 222/666 and 296/888

The average score for image quality was $3.3 \pm 0.7$ for the entire cohort. Image quality was significantly worse for the 296/888MBq group when compared to the 111/333MBq group, but otherwise there was no significant difference in image quality between the dosing strategies. Image quality for exercise studies was significantly better, or trended towards significance, when compared to pharmacological studies, with the exception of the 222/666MBq group. Image quality details are shown in Figure 3. Representative images for the 111/333MBq dosing strategy are shown in Figures 4a, 4b, and 4c. 


\section{Image Quality}

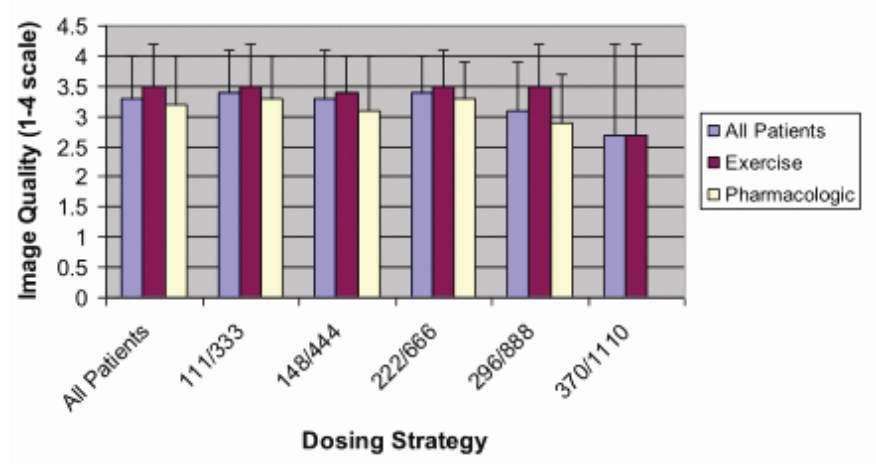

Studies graded on a 1-4 scale, with 1=poor, 2=fair, 3=good, and 4=excellent

Figure 3. Image Quality Comparison by Dosing Strategy

Figure 3. Statistically significant differences include: All Patients: 111/333 vs. 296/888. Pharmacologic Group: 111/333 vs. 296/888. Exercise vs. Pharmacologic Comparison: The whole exercise cohort vs. the whole pharmacologic cohort, and the111/333 exercise compared to 111/333 pharmacological

Image quality was reassessed in a subset (10\%) of the studies to evaluate inter-observer variability. The weighted kappa correlation was 0.489 , which is consistent with moderate inter-observer agreement.

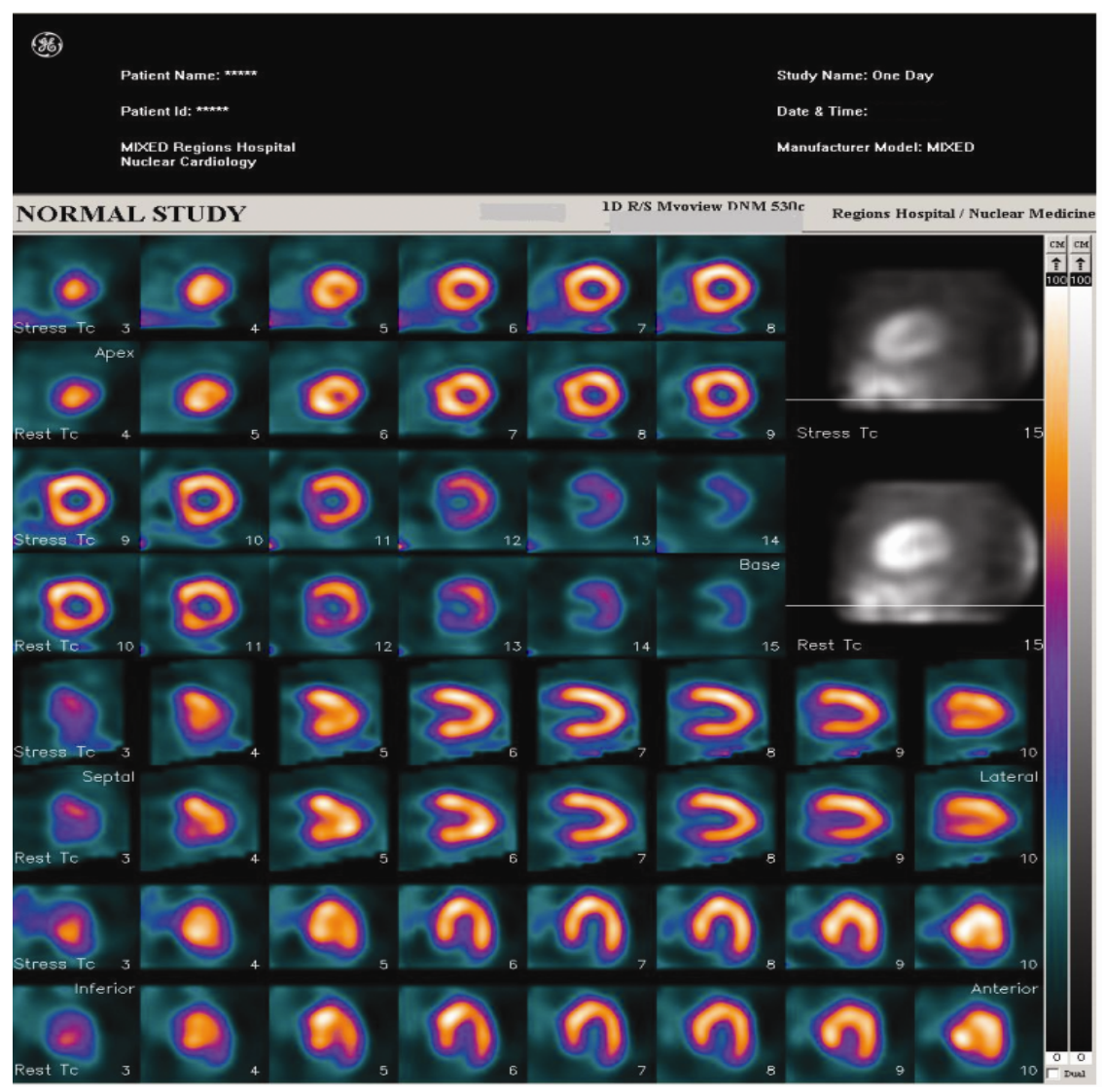

Figure 4a. Normal SPECT MPI study performed at 111/333 MBq (3/9mCi) 


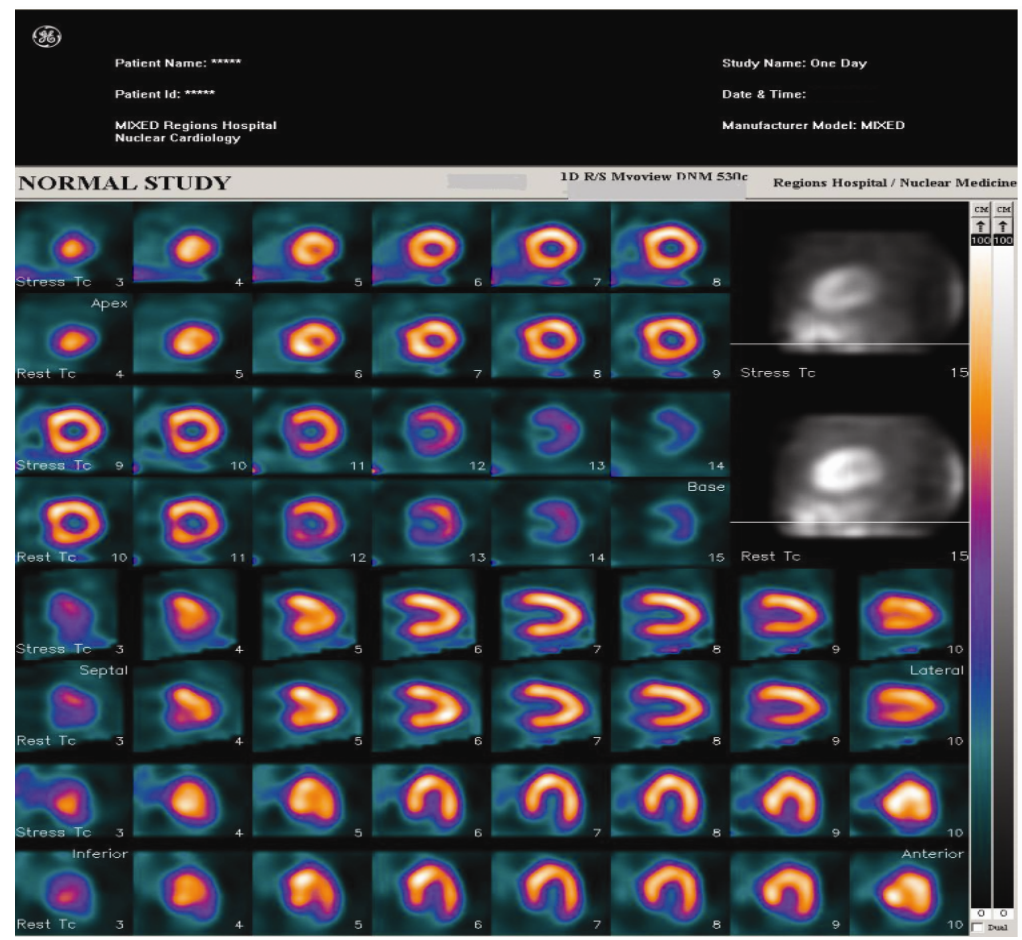

Figure 4b. SPECT MPI study demonstrating inferior ischemia at 111/333 MBq (3/9mCi)

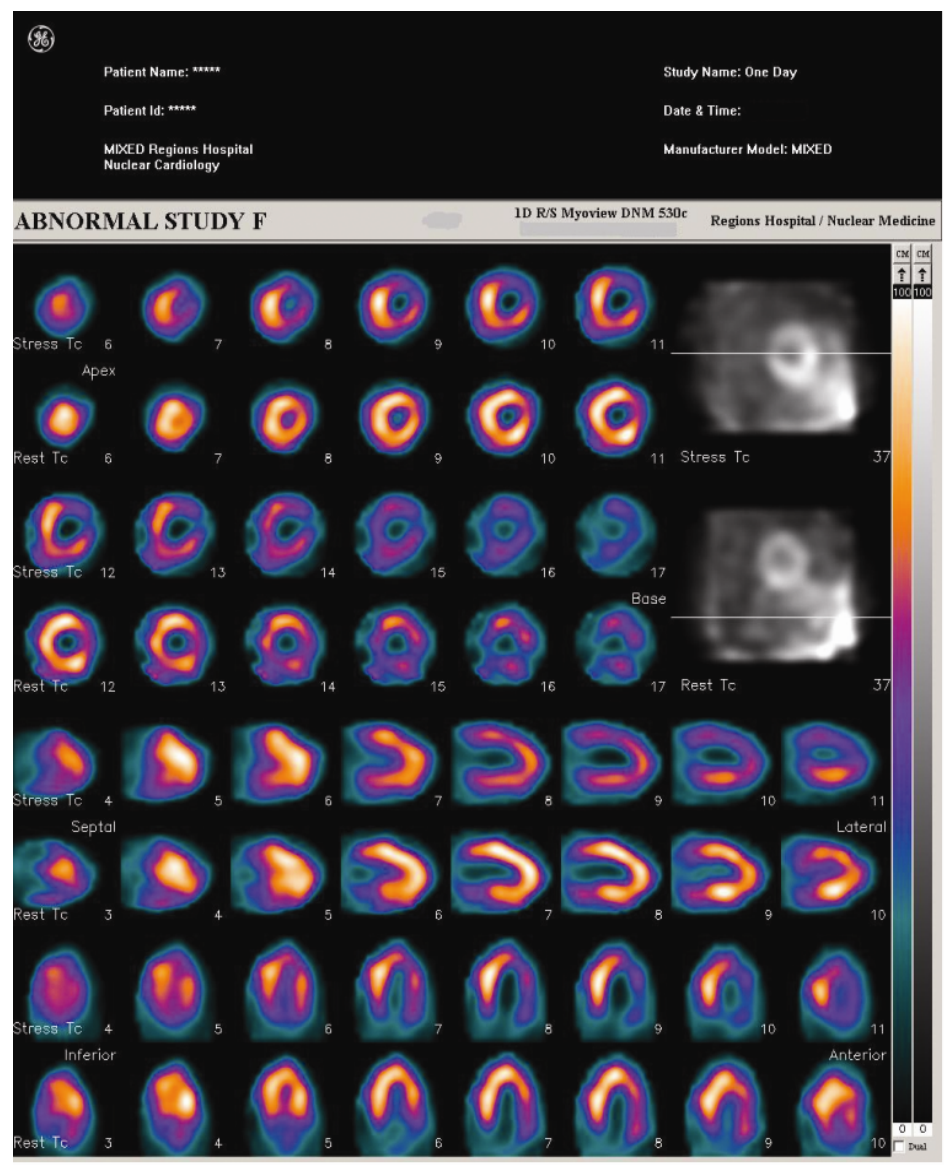

Figure 4c. SPECT MPI study demonstrating anterior ischemia at 111/333MBq (3/9mCi) 


\section{Angiographic correlation}

The majority of scans were normal (65\%) (267/414), while 30\% (124/414) were abnormal with either ischemia or infarction identified. A small percentage of scans (5\%) (22/414) were considered equivocal by the reading cardiologist.

There were 35 patients (8\%) who were referred for angiography after their MPI study. All decisions and interpretations made at the time of angiography were clinically based, and made at the discretion of the interventional cardiologist.

Patients proceeded to angiography due to the following reasons: ischemia present on MPI study (69\%) (24/35) continued symptoms or clinical decision despite negative MPI results (20\%) (7/35), new infarct identified on MPI study (8\%) (3/35), or non-ST elevation myocardial infarction (3\%) (This patient presented 35 days after SPECT MPI, which had demonstrated a large infarct without ischemia). Angiography revealed a sensitivity of $96 \%$, specificity of $50 \%$, positive predictive value of $87 \%$, negative predictive value of $80 \%$, with an accuracy of $86 \%$ for detecting coronary artery disease. The $2 \times 2$ table includes 30 patients with disease predicted. Four of these were false positives, with no obstructive disease demonstrated. Twenty-one had a coronary intervention performed in a vascular territory that correlated with the ischemic region demonstrated on SPECT. Five had a new infarct demonstrated on perfusion imaging, and underwent intervention performed in an artery corresponding to the infarcted territory. The remaining five patients had no ischemia or infarct demonstrated on perfusion imaging. One of these five was found to have an obstructive coronary lesion.

\section{Discussion}

Reducing radiation levels associated with medical diagnostic imaging has become a topic of increased interest and concern in the general population and medical community, with several organizations, including the Food and Drug Administration, American College of Cardiology, American Heart Association, and the American Society of Nuclear Cardiology, releasing recommendations on enhanced radiation safety ${ }^{[7-9]}$. SPECT MPI is traditionally associated with radiation doses in the $10-20 \mathrm{mSv}$ range ${ }^{[3]}$. The goal recently established by the American Society of Nuclear Cardiology is to reduce the radiation exposure to $<9 \mathrm{mSv}$ for $50 \%$ of nuclear cardiology exams by $2014{ }^{[9]}$. Recent publications and abstracts have identified hardware and software advancements that have resulted in substantial reductions in radiation doses, as well as shortened image acquisition times ${ }^{[10,11]}$.

The use of CZT technology, with a higher photon flux than standard sodium-iodide scintillation gamma camera imaging, allows for the reduction in image time and/or dose of radiopharmaceutical. We have demonstrated in this cohort of patients a reduced imaging time, as well as a substantial reduction in radiation dose, with an average expected radiation dose of $4.8 \mathrm{mSv}$ for the 414 patients in this cohort. The majority of the patients in our study (58\%) received expected radiation doses of only 3.6mSv. Only $7 \%$ of our studies had expected radiation doses that exceeded the $9 \mathrm{mSv}$ threshold identified by the American Society of Nuclear Cardiology, with the remaining 93\% of studies falling well below this level. This easily surpasses the 2014 goal set forth by the American Society of Nuclear Cardiology for radiation exposure ${ }^{[9]}$, with the majority of patients receiving less than $4 \mathrm{mSv}$. We believe this to be the lowest reported radiation dose for rest/stress MPI in a clinical setting.

Even further reductions in radiation dose may be possible. Coupling CZT technology with new advances in iterative reconstruction software could provide an opportunity for further reductions in radiation exposure ${ }^{[10,11]}$. Moreover, none of the patients in our cohort had stress-only MPI, another method for dose reduction. It has been shown that low-dose, stress-only MPI can also significantly reduce radiation exposure, and with expected radiation doses below $2 \mathrm{mSv}$ being reported ${ }^{[11]}$.

Similar to previous publications, about two-thirds of our SPECT MPI studies were normal (65\%), with the remaining one-third being abnormal (30\%), and a small percentage (5\%) being equivocal ${ }^{[4,6]}$. Angiographic correlation in a subset of our cohort found an excellent sensitivity (96\%), and high degree of accuracy (86\%) for detecting coronary disease, which 
is consistent with previous publications ${ }^{[4]}$. The specificity of $50 \%$ is also consistent with previous publications on SPECT MPI ${ }^{[4]}$. This specificity may not be entirely representative of the true specificity associated with SPECT MPI, as patients with normal studies rarely proceed on to angiography, thus limiting a more robust and accurate evaluation of true negative and false negative studies ${ }^{[4]}$.

This low-dose protocol has shortened the rest/stress scan times, which results in improved patient satisfaction. It has also allowed us to negotiate a cost savings with our radiopharmaceutical provider, as the decreased doses significantly reduce our isotope demand. The cost of the isotope for the 414 patients in our cohort would have cost approximately \$8,000 more following protocols preceding the implementation of this low-dose strategy. We've continued to benefit from our reduced isotope demand, and our current savings approach $\$ 100,000$ per year from our radiopharmaceutical provider. Lastly, this low-dose protocol has improved patient safety by significantly reducing the radiation exposure for the patient.

\section{Limitations}

The results of this study are from a single center. Image quality was assessed by eight different nuclear cardiologists. The effect of intra-observer variability was assessed, with a weighted kappa correlation coefficiency, which found moderate agreement between the respective readers and one expert reader. There are no comparisons between our low-dose imaging and more traditional dose imaging within the same body weight or BMI group.

\section{Conclusion}

CZT technology permits rapid, very low-dose MPI studies to be performed, significantly reducing radiation exposure and improving patient experience, while still providing high quality, diagnostic perfusion results. The majority of our patients were imaged with a rapid protocol at less than $4 \mathrm{mSv}$. We believe this to be the lowest reported clinically used rest/stress protocol.

\section{References}

[1] Jaarsma C, Leiner T, Bekkers SC, Crijns HJ, Wildberger JE, Nagel E, et al. Diagnostic performance of noninvasive myocardial perfusion imaging using single photon-emission computed tomography, cardiac magnetic resonance, and positron emission tomography imaging for the detection of obstructive coronary artery disease: a meta-analysis. J Am Coll Cardiol. 2012; 59: 1719-1728. http://dx.doi.org/10.1016/j.jacc.2011.12.040

[2] Metz LD, Beattie M, Hom R, Redberg RF, Grady D, Fleischmann KE. The prognostic value of normal exercise myocardial perfusion imaging and exercise echocardiography: a meta-analysis. J Am Coll Cardiol. 2007; 49: 227-237. http://dx.doi.org/10.1016/j.jacc.2006.08.048

[3] Einstein AJ, Moser KW, Thompson RC, Cerqueira MD, Henzlova MJ. Radiation dose to patients from cardiac diagnostic imaging. Circulation. 2007; 116: 1290-1305. http://dx.doi.org/10.1161/CIRCULATIONAHA.107.688101

[4] Duvall WL, Sweeny JM, Croft LB, Barghash MH, Kulkarni NK, Guma KA, et al. Comparison of high efficiency CZT SPECT MPI to coronary angiography. J Nuc Cardiol. 2011; 18: 595-604. http://dx.doi.org/10.1007/s12350-011-9382-z

[5] Duval WL, Croft LB, Godiwala T, Ginsberg E, George T, Henzlova MJ. Reduced isotope dose with rapid SPECT MPI imaging: initial experience with a CZT SPECT camera. J Nuc Cardiol. 2010; 17: 1009-1014. http://dx.doi.org/10.1007/s12350-010-9215-5

[6] Duvall WL, Croft LB, Ginsberg ES, Einstein AJ, Guma KA, George T, et al. Reduced isotope dose and imaging time with a high-efficiency CZT SPECT camera. J Nuc Cardiol. 2011; 18: 847-857. http://dx.doi.org/10.1007/s12350-011-9379-7

[7] Douglas PS, Carr JJ, Cerqueira MD, Cummings JE, Gerber TC, Mukherjee D, et al. Developing an action plan for patient radiation safety in adult cardiovascular medicine: proceedings from Duke University Clinical Research Institute/American College of Cardiology Foundation/American Heart Association. J Am Coll Cardiol. 2012; 59: 1833-1847.

http://dx.doi.org/10.1016/j.jacc.2012.01.005 
[8] United States Food and Drug Administration Public Meeting: Device improvements to reduce unnecessary radiation exposure from medical imaging. 2010 March; 30-31. Available from: http://www.fda.gov/medicaldevices/newsevents/workshopsconferences/ucm201448.htm.

[9] Cerqueira MD, Allman KC, Ficaro EP, Hansen CL, Nichols KJ, Thompson RC, et al. Recommendations for reducing radiation exposure in myocardial perfusion imaging. J Nucl Cardiol. 2010; 17: 709-718. http://dx.doi.org/10.1007/s12350-010-9244-0

[10] DePuey EG. Advances in SPECT camera software and hardware: currently available and new on the horizon. J Nucl Cardiol. 2012; 19: 551-581. http://dx.doi.org/10.1007/s12350-012-9544-7

[11] Zafrir N. Feasibility of myocardial perfusion imaging with radiation dose reduction by half. International Conference of Non-Invasive Cardiovascular Imaging; 2011 May; 17:196. Amsterdam, Netherlands 